\section{Biomarkers in localized prostate cancer}

\author{
Matteo Ferro ${ }^{\ddagger 1}$, Carlo Buonerba ${ }^{*, \neq, 2}$, Daniela Terracciano ${ }^{3}$, Giuseppe Lucarelli ${ }^{4}$, \\ Vincenzo Cosimato ${ }^{3}$, Danilo Bottero', Victor M Deliu', Pasquale Ditonno ${ }^{4}$, \\ Sisto Perdonà ${ }^{5}$, Riccardo Autorino ${ }^{6}$, Ioman Coman7, Sabino De Placido², \\ Giuseppe Di Lorenzo² \& Ottavio De Cobelli ${ }^{1,7}$
}

Biomarkers can improve prostate cancer diagnosis and treatment. Accuracy of prostatespecific antigen (PSA) for early diagnosis of prostate cancer is not satisfactory, as it is an organbut not cancer-specific biomarker, and it can be improved by using models that incorporate PSA along with other test results, such as prostate cancer antigen 3 , the molecular forms of PSA (proPSA, benign PSA and intact PSA), as well as kallikreins. Recent reports suggest that new tools may be provided by metabolomic studies as shown by preliminary data on sarcosine. Additional molecular biomarkers have been identified by the use of genomics, proteomics and metabolomics. We review the most relevant biomarkers for early diagnosis and management of localized prostate cancer.

First draft submitted: 6 August 2015; Accepted for publication: 11 November 2015; Published online: 15 January 2016

The European randomized study of screening for prostate cancer showed that prostate-specific antigen (PSA)-based screening in senior men was associated with a statistically significant $29 \%$ prostate cancer mortality reduction [1]. Nevertheless, as many as $40 \%$ of those screened are at risk to be treated for a biologically indolent disease that will not affect their life expectancy [1], which results in a $23 \%$ negative impact on the life-years gained [2]. The conclusion of one recently published cost-effectiveness analysis was that PSA-based screening should be limited to two or three screens between ages 55 and 59 years because in men older than 63 years overdiagnosis is responsible for loss of quality-adjusted life-years [3]. Several clinical, pathological and biological variables [4] were reported to improve the modest accuracy of PSA as a screening test, although their impact in a community-based setting is yet to be assessed in large trials. An analysis of six externally validated PSA-based models that incorporated readily available variables such as age, race, digital rectal examination, previous biopsies, family history, free PSA, transrectal ultrasonography prostate volume showed that five of these were able to double the sensitivity of PSA testing (44 vs 21\%) without loss of specificity [5]. Furthermore, the Prostate Cancer Prevention Trial model can also predict the presence of Gleason $\geq 7$ versus Gleason $<7$ prostate cancer [6]. Many novel biomarkers also provide valuable prognostic information that can have important therapeutic implications and serve as

'Division of Urology, European Institute of Oncology, Milan, Italy

${ }^{2}$ Medical Oncology, Department of Clinical Medicine \& Surgery, University 'Federico II', Naples, Italy

${ }^{3}$ Department of Translational Medical Sciences, University 'Federico II', Naples, Italy

${ }^{4}$ Department of Emergency \& Organ Transplantation - Urology, Andrology \& Kidney Transplantation Unit, University of Bari, Bari, Italy

${ }^{5}$ Department of Urology, National Cancer Institute of Naples, Naples, Italy

${ }^{6}$ Urology Institute, University Hospitals Case Medical Center, Cleveland, OH 44106, USA

'Department of Urology 'Iuliu Hatieganu', University of Medicine \& Pharmacy, 400012 Cluj-Napoca, Romania

*Author for correspondence: carbuone@hotmail.com

${ }^{\ddagger}$ Authors contributed equally

\section{KEYWORDS}

- biomarkers • prostate cancer 
selection criteria for patients eligible for active surveillance or candidates for radiotherapy/surgery [7]. In this review, we will focus on the most relevant biomarkers potentially useful for prostate cancer early diagnosis and for assessment of the prognosis of localized disease. Evidence supporting the use of the biomarkers discussed is presented along with a critical analysis of their potential impact on future research and clinical practice.

\section{Serum biomarkers}

\section{- Prostate-specific antigen}

PSA is a glycoprotein serine protease that promotes sperm motility via degradation of the semenogelin I and II proteins [8]. In a landmark study conducted by Catalona [9] and colleagues involving 1653 healthy men, the combined use of serum PSA measurement with a positive cutoff of $4 \mathrm{ng} / \mathrm{ml}$ in combination and digital rectal examination, with ultrasonography performed in case of abnormal findings, yielded significantly better accuracy than rectal examination alone. The inexpensiveness and the noninvasiveness of PSA testing made it widely available as a screening tool for prostate cancer, in spite of its unsatisfactory specificity [10]. Serum PSA can also rise in several nonmalignant prostatic diseases, such as benign prostatic hyperplasia [11], which shows a histological prevalence at autopsy of $50 \%$ in men aged $50-60$ years and of $90 \%$ in men over 80 years old [12]. Conversely, among 2950 men enrolled in the Prostate Cancer Prevention Trial showing PSA levels lower than or equal to $4 \mathrm{ng} / \mathrm{ml}$ and normal digital rectal examination, prostate cancer was detected in 449 patients $(15.2 \%)$, with 67 of them showing a Gleason score of 7 or higher. The specificity and sensitivity of PSA alone ranges from 20 to $40 \%$ and 70 to $90 \%$, respectively, while the AUC metric of the receiver operating characteristic curve is 0.55 and 0.70 for the accuracy, depending on the cutoff values employed (e.g., 4 vs 3 ng/ml). PSA positive predictive value is only $25-40 \%$ [13]. Furthermore, PSA levels are dependent on age and race. In one study assessing a total of 541 evaluable prostate cancer patients (408 white and 133 black) with pretreatment PSA assessment, the mean PSA value for black men was $14.00 \mathrm{ng} / \mathrm{ml}$ compared with $8.29 \mathrm{ng} / \mathrm{ml}$ for white men $(\mathrm{p}<0.001)$. This difference remained statistically significant after adjusting for stage, grade and age. Black men also had tumor volumes 1.3-2.5-times greater than those of white men, depending on tumor stage [14]. In another study evaluating 471 men without evidence of prostate cancer serum PSA concentration was correlated with patient age $(\mathrm{r}=0.43 ; \mathrm{p}<0.0001)$ and prostatic volume $(\mathrm{r}=0.55 ; \mathrm{p}<0.0001)$, with mean serum PSA concentration increasing by approximately $0.04 \mathrm{ng} / \mathrm{ml}$ per year in a healthy 60-year-old man [15]. In view of these findings, age-specific total PSA cutoff shave has been suggested in order to account for the physiological age-dependant increase in prostatic volume [15], and the American Urology Association recommended to discontinue PSA screening in men older than 70 years with a PSA below $3 \mathrm{ng} / \mathrm{ml}$ [16]. PSA levels were also associated with polymorphisms of the PSA promotor and of the kallikrein gene, among other genes. In one study involving 409 healthy white men, the analysis of the promoter region of the PSA genes showed that each of the $-4643 \mathrm{G} / \mathrm{A}$, $-5412 \mathrm{C} / \mathrm{T}$ and $5429 \mathrm{~T} / \mathrm{G}$ single nucleotide polymorphisms were reported in approximately $20 \%$ of the population and were significantly associated with increases in serum PSA levels and PSA promoter activity. In another study conducted in 303 male military conscripts, PSA levels were found to be strongly associated with the rs1058205 kallikrein polymorphism [17]

Given the limitations of PSA testing alone, a number of PSA derivatives have been investigated in order to improve PSA diagnostic accuracy. PSA density is computed by dividing PSA by prostate volume and is a strong predictor of adverse pathological features and biochemical recurrence after radical treatment $[18,19]$. Furthermore, PSA density can be particularly useful for the evaluation of patient candidates for active surveillance, since it is associated with Gleason score upgrade in patients with biopsy Gleason score of 6 and $3+4=7[20,21]$. In this regard, it must be noted that PSA density is an established prognostic factor and serves as an inclusion criterion for active surveillance [22]

Measures of PSA kinetics, such as PSA velocity (PSAV) and PSA doubling time, were predictive of biopsy results in patients with suspicion of prostate cancer and disease recurrence and survival after radical treatment, although it is not clear whether these measures can improve the accuracy of models based on PSA only [23,24]. One study conducted in 32 patients with high-risk prostate cancer, 606 men without prostate cancer and 79 patients with prostate cancer who were alive or dead of another 
cause, assessed the PSAV risk count, defined as the number of times PSAV exceeded a certain threshold. A higher PSAV risk count was significantly associated with the onset of high-risk prostate cancer after adjusting for known predictors (relative risk: $1.41 ; 95 \% \mathrm{CI}: 1.25-1.59$ for a PSAV cutpoint $0.2 \mathrm{ng} / \mathrm{ml} /$ year; relative risk: 1.49; 95\% CI: $1.29-1.71$ for a PSAV cutpoint of $0.4 \mathrm{ng} / \mathrm{ml} /$ year; $\mathrm{p}<0.001$ ) [25]. Moreover, a PSAV risk count of 2 was associated with a significantly higher risk of aggressive disease in men undergoing biopsy after accounting for age and PSA [26], while in another study a risk count of 3 or 2 was associated with a significantly increased risk of diagnosis of high-grade disease on re-biopsy in a surveillance cohort [27]. The PSAV risk count has the potential to overcome the limitation associated with PSA kinetics due to the physiologic fluctuations of PSA.

\section{- Prostate health index}

'Free PSA' is a part of circulating PSA that is not protein bound and it had been reported as a promising biomarker of biopsy and re-biopsy findings $[26,28-30]$ before subsequent studies failed to show such an association [31,32]. Precursor forms of PSA (pPSA) are a component of free PSA and were detected at higher levels in prostate cancer tissue with respect to benign prostatic hyperplasia or healthy prostate tissue [33]. In a pioneering study conducted in five patients with prostate cancer and three controls, the truncated form of PSA precursor, namely [-2]pPSA, was estimated to range from 25 to $95 \%$ of the free PSA in prostate cancer patients but only $6-19 \%$ of the free PSA in the biopsy-negative men [34]. [-2]pPSA presents a serine-arginine pro leader peptide and is sufficiently stable to be measured reliably using the Beckman Coulter assay in order to compute phi by using the formula ([-2]proPSA/free PSA) $\times \sqrt{ }$ PSA [35]. In one study including 736 serum samples of patients screened for prostate cancer, the use of phi yielded a significantly better accuracy, with an AUC of 0.71-0.75, with respect to PSA (AUC $=0.53-0.58$ ) and \%free PSA (AUC $=0.57-0.67)$. Also \%p2PSA showed a better accuracy than PSA and \%free PSA [35]. In men aged 50 years and older with a negative digital rectal examination and a serum PSA between 4 and $10 \mathrm{ng} / \mathrm{ml}$ phi and\%[-2]proPSA have the highest accuracy for the detection of cancer of any grade [36]. Furthermore, phi and $\%[-2]$ proPSA are useful tools for the diagnosis of Gleason $\geq 7$ prostate cancer and outperform
PSA and free PSA in men with a positive family history [37] and in men with PSA levels between 2 and $10 \mathrm{ng} / \mathrm{ml}$ [38]. In an analysis of $158 \mathrm{men}$ with a first-degree relative with prostate cancer, p2PSA,\%p2PSA and phi values were significantly higher in 71 men with prostate cancer $(44.9 \%)$ than in those without prostate cancer. At multivariable analysis, \%p2PSA and phi were independent predictors of positive biopsy and significantly improved the accuracy of PSA and prostate volume by 8.7 and $10 \%$, respectively [37]. The diagnostic accuracy of phi was confirmed by a meta-analysis performed by Bruzzese and colleagues in 2969 men undergoing first biopsy with PSA serum levels in the range of 2-10 ng $/ \mathrm{ml}$. Biopsy-confirmed prostate cancer was detected in $1287(43.3 \%) \mathrm{men}$. The AUC of phi and\%fPSA were 0.74 (95\% CI: 0.70-0.77) and 0.63 (95\% CI: $0.58-0.67$ ), respectively, with a superiority of phi compared with \%fPSA and a relative diagnostic odds ratio of 2.81 (95\% CI: 2.19-3.6; $\mathrm{p}<0.0001)$ [39]

\section{- $4 \mathrm{~K}$ score}

The four kallikrein panel, namely the $4 \mathrm{~K}$ score, is an extensively investigated test that is based on four kallikrein blood markers, that is total PSA, free PSA, intact PSA and the human kallikreinrelated peptide 2 . In a series of 161 men undergoing prostatectomy, serum levels of human glandular kallikrein 2 were significantly higher in patients with organ versus nonorgan-confined disease [40]. In a study [41] involving 1501 men with elevated PSA, the $4 \mathrm{~K}$ score showed a significantly higher predictive accuracy for cancer than a model based on PSA, age and digital rectal examination only, with an AUC of 0.71 versus 0.58 . These results were consistent with those obtained in an analysis of 11,063 Swedish men enrolled in the Malmö Diet and Cancer study during 1991-199, which showed that the $4 \mathrm{~K}$ test predicted a subsequent diagnosis of clinically significant cancer, with improved concordance index compared with total PSA and age ( 0.75 vs $0.65 ; \mathrm{p}<0.001)$ [41]. Several studies showed that the $4 \mathrm{~K}$ test is able to reduce the number of biopsies at the cost of missing only few high-grade cancers $[42,43]$. One study conducted in 262 men undergoing prostate biopsy because of elevated PSA ( $\geq 3 \mathrm{ng} / \mathrm{ml}$ ) showed that the $4 \mathrm{~K}$ test was able to reduce biopsies by approximately $50 \%$, with only $1 \%$ of aggressive cancer diagnoses missed [44]. Similar findings were obtained in another cohort of 740 screened men undergoing biopsy for elevated PSA [11]. In 
a cohort of 925 men with negative findings on a previous biopsy, the use of the $4 \mathrm{~K}$ test improved the accuracy of a model based on PSA and digital rectal examination alone $(\mathrm{AUC}=0.68$ vs 0.58 for any cancer and AUC $=0.87$ vs 0.76 for highgrade cancer) [41]. Finally, a prospective evaluation of the $4 \mathrm{~K}$ score as a predictive factor of Gleason $\geq 7$ prostate cancer was conducted in 1012 men scheduled for prostate biopsy and showed that the $4 \mathrm{~K}$ score presented higher discrimination $(\mathrm{AUC}=0.82)$ and net benefit compared with a modified Prostate Cancer Prevention Trial Risk Calculator 2.0 model, with a possible reduction of $30-58 \%$ in the number biopsies and delayed diagnosis in only $1.3-4.7 \%$ of Gleason $\geq 7$ prostate cancer cases [45]. Finally, in one study involving 1423 cases of prostate cancer, with 235 patients with distant metastasis among men with PSA $>2 \mathrm{ng} / \mathrm{ml}$, a prespecified model based on four kallikrein markers significantly enhanced the prediction of metastasis compared with PSA alone [46]. Overall, there is sufficient evidence to consider the $4 \mathrm{~K}$ score as a valuable diagnostic and prognostic tool for the management of early prostate cancer.

\section{- miRNAs}

miRNAs are small, noncoding RNAs with important functions as regulators of gene expression at the post-transcriptional level [47]. Both tissue and circulating miRNAs have the potential to be employed as diagnostic and prognostic markers for various cancers including prostate cancer [48]. Similarly to mRNA [48], miRNA expression signatures in prostate cancer tissues predict both Gleason score and prognosis of localized prostate cancer [49-51]. Such information is remarkably useful in order to avoid overtreatment.

Mahn et al. [52] studied a sample including 37 prostatectomy patients with localized prostate cancer, 18 patients with benign prostate hyperplasia treated with retropubic adenomectomy or transurethral resection of the prostate, eight patients with metastatic prostate cancer as well as 20 healthy volunteers. Four oncogenic miRNAs (miR-32, miR-26a, miR-let7i and miR-195) plus miR-16 were assessed in the serum of men included in the study sample. For the differential diagnosis of benign prostate hyperplasia versus prostate cancer, the use of miR-26a alone level was moderately accurate, with an AUC of 0.703, while the combined use of oncogenic miRNAs was associated with an AUC of 0.758. Of note, the Gleason score was associated with miR-195 and miR-let7i expression levels. In a study sample of 82 prostate cancer patients, Shen et al. [53] showed that prostate cancer patients with highrisk CAPRA scores had higher miR-20a and miR-21 levels. Furthermore, miR-21 and miR221 levels were able to differentiate patients with low versus intermediate risk CAPRA scores $(A U C=0.801 ; p=0.002)$. These findings confirm that miRNAs are associated with different levels of cancer aggressiveness in prostate cancer patients.

\section{Urine biomarkers}

\section{- Sarcosine (N-methyl glycine)}

Metabolomic profiling is a powerful tool for the comprehensive analysis of the complete set of metabolic intermediates in normal and cancer cells. This approach can be used to characterize the metabolic fingerprint of a tumor and identify novel markers that may be used for prostate cancer early detection, prognostic stratification and therapy response monitoring [54].

In a proof-of-concept study published in 2009, Sreekumar et al. explored the prostate cancer metabolome [55]. This was characterized by an increased amino acid metabolism and a perturbation of nitrogen breakdown pathways, along with high total choline-containing compounds and phosphocholine levels. In addition, androgens had an important role in regulating the prostate cancer amino acid metabolism, and in altering the methylation potential, in accordance with the increased expression of methyltransferases like EZH2 [56].

Among the perturbed metabolites, sarcosine - an N-methyl derivative of glycine - was significantly increased during prostate cancer progression from normal through localized to metastatic disease [55]. Interestingly, the analysis of patients with PSA levels in the range 2-10 ng/ml, showed that urinary sarcosine had a higher predictive value than PSA in differentiating prostate cancer patients from negative controls. Sreekumar et al. [55] validated their results in an independent cohort of patients and confirmed their previous results of increased levels of urinary sarcosine in prostate cancer patients. Cao et al. evaluated sarcosine levels in urine supernatants and sediments and used prostate cancer antigen 3 (PCA3) and free PSA as comparators. Urinary sarcosine was significantly higher in prostate cancer patients than in controls, and when this marker was combined with PCA3 or free PSA, the combined model had a higher predictive value [57]. 
Further studies demonstrated that serum sarcosine had a higher predictive value than total PSA and free PSA in detecting prostate cancer in patients with total serum PSA $<4 \mathrm{ng} / \mathrm{ml}$. Serum sarcosine had the largest AUC in predicting lowgrade, low-PSA prostate cancer, suggesting that this biomarker may be a further tool not only for diagnosis, but also for selecting patients for active surveillance strategies [58-60].

In addition, elevated circulating sarcosine levels have been showed in patients with metastatic castration-resistant prostate cancer compared with patients with nonmetastatic disease [61]. In particular, Kaplan-Meier curve analysis demonstrated differences in overall and progression-free survivals between patients with high versus low sarcosine values. At multivariate analysis, this metabolite remained an independent prognosticator for overall and progression-free survivals. Interestingly, a significant correlation resulted between serum sarcosine levels and the duration of hormone sensitivity. This finding can be explained by considering that glycine-N-methyltransferase gene - that encodes for enzymes that generate sarcosine by transferring a methyl group from $\mathrm{S}$-adenosylmethionine to glycine - has binding sequences for androgen receptor and ETS transcription factor family members. Therefore, these findings directly link activation of the sarcosine pathway to androgen receptor and TMPRSS2:ETS gene fusion regulation [61]. In the PLCO trial, a positive association was identified between elevated serum sarcosine and prostate cancer. The results of this large prospective study suggested that serum sarcosine could be a potential biomarker for early prostate cancer detection, specifically for nonaggressive disease, and showed a stronger association between sarcosine levels and prostate cancer risk in men with diabetes [62].

\section{- PCA3 score}

The DD3 (PCA3) gene is a noncoding RNA mapped to chromosome 9q21-22 that was originally found to be highly specific for prostate cancer tissue with respect to normal prostatic tissue [63]. PCA3 was expressed only in the LNCaP cell line among several human prostate cancer cell lines tested including ALVA-31, DU-145, JCA-1, LNCaP, PC-3, PPC-1 and TSU-pr1. While PCA3 is significantly expressed in androgen receptor positive prostate cancer cells, it is expressed at very low levels in the adjacent non-neoplastic tissue and benign prostate hyperplasia cells [64].
A meta-analysis evaluated the accuracy of PCA3 in predicting re-biopsy outcome in two subgroups respectively including patients with and without either atypical small acinar proliferation or high-grade prostatic intraepithelial neoplasia. If a PCA 3 cutoff of 20 or 35 was used, the overall sensitivity values were 0.93 or 0.80 and 0.79 or 0.75 , while the specificity values were 0.65 or 0.44 and 0.78 or 0.70 , respectively, in the two subgroups. The AUCs of the summary receiver operating characteristic curve were 0.85 or 0.72 and 0.81 or 0.69 , respectively. These findings confirm that thePCA3 score is able to avoid unnecessary biopsies by using a cutoff score of 20 [65].

\section{TMPRSS2:ERG gene fusion}

The TMPRSS2:ERG gene fusion is a frequent chromosomal rearrangement in prostate cancer. It is the result of the fusion of the TMPRSS 2 gene and the v-ets avian erythroblastosis virus E26 oncogene homolog $(E R G)$ gene or other ETS (E26 transformation specific) transcription factors. The TMPRSS2:ERG gene fusion is regarded as one of the most frequent gene-specific alterations in prostate cancer [66].

Similarly to PCA3, a TMPRSS2:ERG rearrangement can be detected in urine after digital rectal examination [66] and can also be normalized to the amount of PSA mRNA in order to compute a TMPRSS2:ERG score. TMPRSS2:ERG is highly specific for predicting clinically significant prostate cancer on biopsy, despite the relatively low sensitivity. Gene fusion test can correctly detect cancer in 50-75\% of cases [67,68].

Robert et al. provided a rational basis for combining PCA3 and TMPRSS2:ERG in tissue samples [69]. After the first study on combining PCA3 and TMPRSS2:ERG reported by Hessels et al. [66], several studies [70-72] showed better accuracy of the combination with TMPRSS2:ERG than PCA3 alone for the prediction of prostate cancer detection and progression.

\section{Tissue biomarkers}

\section{- Prolaris}

Prolaris (Myriad Genetics, UT, USA) is a commercially available test that evaluates the expression of 31 cell-cycle progression genes, normalized to 15 housekeeper genes. One study of 413 prostatectomized men reported a hazard ratio for recurrence for each unit increase in the Prolaris score of 1.7 (95\% CI: 1.3-2.4), after adjusting for a standard postoperative risk assessment (Cancer of the Prostate Risk Assessment Postsurgical score) [73,74]. 
Combining the Prolaris score and the Cancer of the Prostate Risk Assessment Postsurgical score improved the concordance index for both the overall cohort and low-risk subset [75]. In one study involving 351 biopsies, the prognostic accuracy of the Prolaris score was evaluated along with other known predictors of survival, such as Gleason score, baseline PSA level, age, clinical stage and extent of the disease. At multivariate analysis, the Prolaris score was associated with a hazard ratio for death of 1.65 (95\% CI: 1.31-2.09) for each unit increase in the Prolaris score, independently on the Gleason score and PSA levels $(p=0.017)$ [74]. In another retrospective study, the Prolaris test was performed in 141 men with prostate cancer treated with curative external beam radiation therapy. With $13 \%$ of patients showing biochemical recurrence, the hazard ratio for biochemical recurrence was 2.55 for a 1-unit increase in the Prolaris score and remained statistically significant even after adjustment for Gleason score, prostate-specific antigen, percent positive cores and androgen deprivation therapy. Furthermore, the Prolaris score was also associated with prostate cancer-specific mortality $(\mathrm{p}=0.013)$ [75].

\section{- Oncotype DX ${ }^{\circledR}$}

The Oncotype DX ${ }^{\circledR}$ Genomic Prostate Score test (Genomic Health, Inc., CA, USA) is a commercially available test that evaluates the expression of 17 genes and is able to provide prognostic information and assess the risk of adverse pathology, defined as primary Gleason pattern 4 or any pattern 5 and/or pT3 disease, after radical prostatectomy. This RT-PCR-based assay was developed and validated in a discovery prostatectomy study involving 441 subjects, in a biopsy study including 167 biopsies, and in a prospective study of 395 patients with low to intermediate clinical risk who were candidates for active surveillance and whose biopsy was tested retrospectively. Of the 732 candidate genes analyzed, 288 (39\%) predicted clinical recurrent disease and 198 (27\%) were associated with aggressive disease after adjusting for clinical stage, prostate-specific antigen and Gleason score. Seventeen genes were finally selected to be included in the Oncotype DX test. In the validation study, the Oncotype DX test significantly predicted both high-grade and high-stage at surgical pathology after controlling for established clinical factors [76]. This assay was performed on biopsies taken from 431 men with very low-, low- or intermediate-risk prostate cancer, defined according to the National Comprehensive Cancer
Network between 1990 and 2011. Oncotype DX was able to predict time to biochemical recurrence at univariable analysis and also after adjusting for the risk group. The test results were also strongly associated with adverse pathology, after adjusting for the risk group [77].

\section{- Methylation assays}

Tumorigenesis is associated with changes in DNA methylation, with hypermethylation involving specific gene promoters in the context of a generalized hypomethylation, thus causing silencing of tumor suppressor genes [78]. A number of genes are hypermethylated in prostate cancer, such as FILIP1L isoform 2 [79], a known senescence marker, the pi-class glutathione S-transferase gene (GSTP1) [80]. In the MATLOC study, histopathologically negative prostate biopsy samples with matching subsequent either positive (cases) or negative (controls) repeat biopsy samples taken within 30 months were analyzed via a quantitative methylation-specific PCR assay panel that included GSTP1, APC and RASSF1. This epigenetic assay yielded a negative predictive value of 90\% (95\% CI: 87-93) [81]. These findings were externally validated in the DOCUMENT study [82], which analyzed cancer-negative prostate biopsy core tissue samples of 350 subjects who did not show (controls) or did show (cases) cancer after repeating biopsy within 24 months. Biopsies were analyzed for GSTP1, APC and RASSF1 relative to the $A C T B$ reference gene by the use of quantitative methylation-specific PCR. The epigenetic assay resulted in a negative predictive value of $88 \%$ (95\% CI: 85-91). As a result, this test appears useful to avoid unnecessary re-biopsy [82] The clinical usefulness of the evaluation of the GSTP1 promoter methylation was confirmed in another study conducted in 42 patients with benign prostatic hyperplasia and in a training and validation cohort of 147 and 71 prostate cancer patients, respectively. The promoter methylation of the APC, CCND2, GSTP1, PTGS2 and RARB genes was evaluated using a quantitative multiplex, methylation-specific PCR assay in formalinfixed, paraffin-embedded tissue samples. Among the genes considered, only GSTP1 methylation was significantly associated with clinical failure in both independent high-risk prostate cancer cohorts [82]. Patients showing either a low or a high GSTP1 methylation level were at a higher risk for clinical failure, both in the training (hazard ratio [HR]: 3.65; 95\% CI: 1.65-8.07) and validation sets (HR: 4.27; 95\% CI: 1.03-17.72) and also in 
Table 1. Summary of biomarker useful in localized prostate cancer.

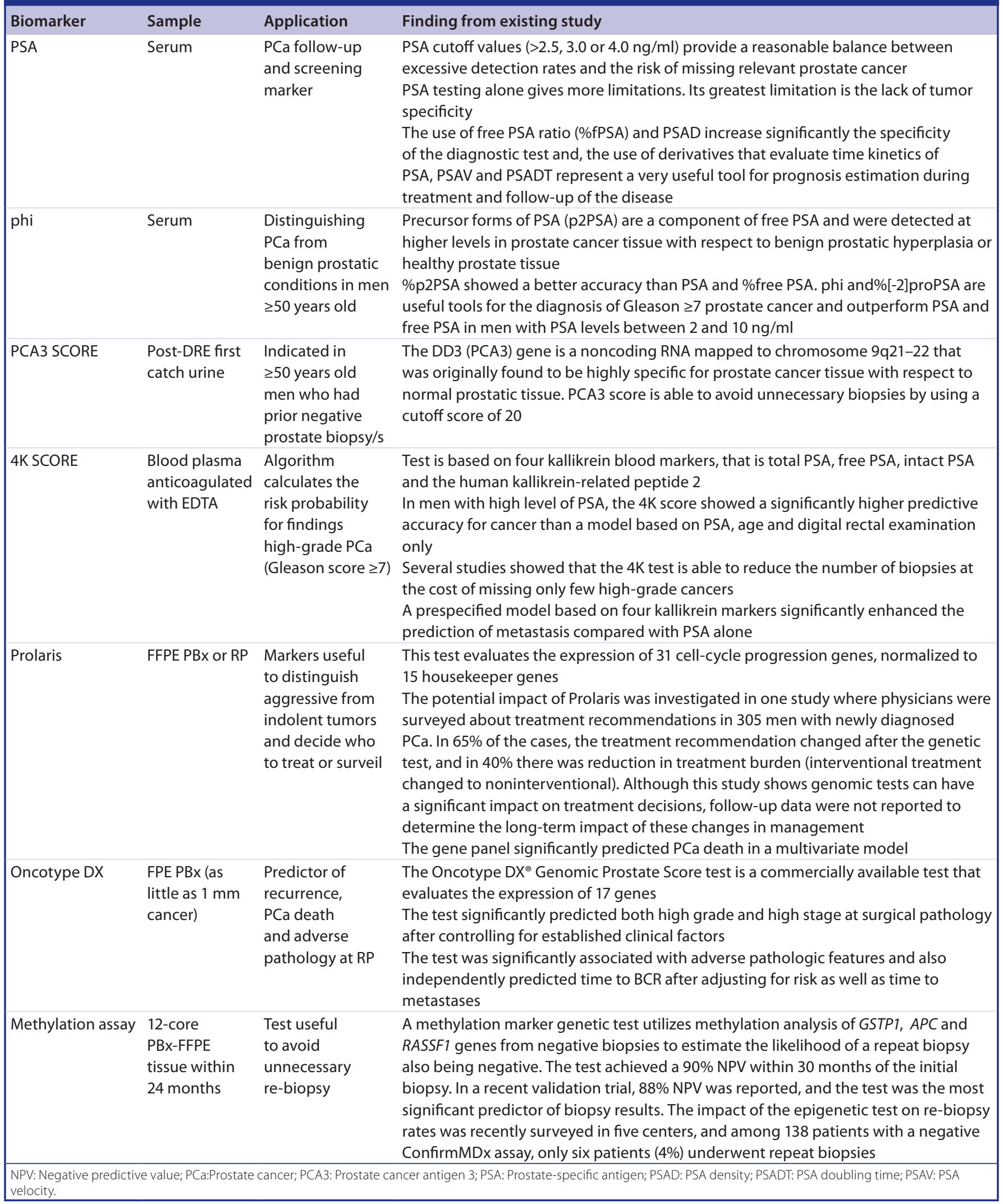


Table 1. Summary of biomarker useful in localized prostate cancer (cont.).

\begin{tabular}{|c|c|c|c|}
\hline Biomarker & Sample & Application & Finding from existing study \\
\hline $\begin{array}{l}\text { Circulating } \\
\text { miRNAs }\end{array}$ & Plasma & $\begin{array}{l}\text { Predict both the } \\
\text { Gleason score and } \\
\text { the relative risk of } \\
\text { PCa lethality }\end{array}$ & $\begin{array}{l}\text { Specific miRNAs are found, not only in tumor tissue, but also in the plasma of PCa } \\
\text { patients; miRNA- } 375 \text { and miRNA-141 are reported to be associated with advanced } \\
\text { PCa. miR-195 correlated with clinic pathological parameters such as surgical margin } \\
\text { positivity and the Gleason score, and that miR-let7i also correlated with some } \\
\text { specific parameters }\end{array}$ \\
\hline $\begin{array}{l}\text { Sarcosine } \\
\text { (N-methylglycine) }\end{array}$ & $\begin{array}{l}\text { Serum and } \\
\text { urine }\end{array}$ & $\begin{array}{l}\text { Evaluation of } \mathrm{PCa} \\
\text { progression }\end{array}$ & $\begin{array}{l}\text { The analysis of patients with PSA levels in the range } 2-10 \mathrm{ng} / \mathrm{ml} \text {, showed that urinary } \\
\text { sarcosine had a higher predictive value than PSA in differentiating PCa patients } \\
\text { from negative controls. When sarcosine was combined with PCA3 or free PSA, the } \\
\text { combined model had a higher predictive value } \\
\text { The serum sarcosine had a higher predictive value than total PSA and free PSA in } \\
\text { detecting PCa in patients with total serum PSA }<4 \mathrm{ng} / \mathrm{ml} \\
\text { Elevated circulating sarcosine levels have been showed in patients with metastatic } \\
\text { castration-resistant PCa compared with patients with nonmetastatic disease }\end{array}$ \\
\hline
\end{tabular}

the combined cohort (HR: 2.74; 95\% CI: 1.425.27), with respect to the moderate methylation groups [83]. In another study involving 149 prostate cancer patients, the promoter methylation of seven genes was evaluated by methylation-sensitive PCR in tissue and urine samples. GSTP1 was confirmed to be frequently methylated in prostate cancer, along with $R A R B$ and $R A S S F 1$ genes. The $R A S S F 1$ gene was methylated in $45 \%$ of prostate cancer urine and was significantly associated with biochemical recurrence in patients at multivariate analysis [84].

The most promising methylation markers recently identified as independent predictors of biochemical recurrence include C1orf114, PITX2, GABRE miR-452 miR-224 and the marker panel AOX1/C1orf114/HAPLN3. All of these candidate biomarkers have been assessed in tissue specimens only, while their noninvasive evaluation (e.g., in circulating tumor cells) is yet to be explored [85].

\section{Conclusion}

Prostate cancer prognosis is highly heterogeneous, with men harboring an indolent disease that can be safely observed and men whose disease is rapidly fatal. The widespread use of PSA as a screening tool has contributed to improve early detection and reduce mortality, but it has also been responsible for overdiagnosis and overtreatment. The complex management of prostate cancer can be improved by the use of diagnostic, prognostic and predictive biomarkers, as discussed in this review and summarized in Table 1.

\section{Future perspective}

Next-generation sequencing-based clinical assays are likely to play an important role as accurate diagnostic tools of localized prostate cancer. Capture exome/transcriptome sequencing on body fluids or biopsy tissues will enable biological discovery useful for precision cancer medicine activities [86]. Whole-exome sequencing has identified genes that are mutated in prostate cancer. This expanded genetic framework may provide new mechanism tools for patient stratification [87]. Recently, Beltran et al. showed the feasibility of performing clinical diagnostics based on deep next-generation sequencing using a novel platform that requires little DNA retrieved from formalinfixed and paraffin-embedded tissue [88]. Of note, the IMPACT screening network supported the use of targeted PSA screening based on $B R C A$ genotype, with a high proportion of aggressive disease [89]. The possibility for the combined use of novel biomarker assays to optimize accuracy is intriguing, but must be weighed against increased costs. There is a compelling need not only for additional well-designed, large, multicenter, prospective trials, but also for economic studies evaluating the cost/benefit ratio of novel biomarkers. Increased attention to reimbursement policies is also required in order to maximize their impact in clinical practice.

\section{Financial \& competing interests disclosure}

The authors have no relevant affiliations or financial involvement with any organization or entity with a financial interest in or financial conflict with the subject matter or materials discussed in the manuscript. This includes employment, consultancies, honoraria, stock ownership or options, expert testimony, grants or patents received or pending, or royalties.

No writing assistance was utilized in the production of this manuscript. 


\section{EXECUTIVE SUMMARY}

\section{Background}

- This review focuses on the most relevant biomarkers potentially useful for prostate cancer early diagnosis and for assessment of the prognosis of localized disease.

\section{Serum biomarkers}

- Among the serum biomarkers/tests considered, prostate-specific antigen (PSA) has a number of limitations for diagnosis and prognostic assessment of localized prostate cancer, but its widespread use is justified because of its inexpensiveness and organ specificity.

- A number of PSA derivatives, such as PSA density, which can be particularly useful for the evaluation of patient candidates for active surveillance, and measures of PSA kinetics, such as PSA velocity, have shown limited usefulness.

- The assessment of PSA-based variables, such as the prostate health index and four kallikrein (4K) panel, has been shown to increase the diagnostic accuracy of the combined use of PSA with clinical variables. As an example, in one study involving 1501 men with elevated PSA, the 4K score showed a significantly higher predictive accuracy for cancer than a model based on PSA, age and digital rectal examination only, with an AUC of 0.71 versus 0.58 .

- The use of miRNA has also been proven useful for the differential diagnosis of prostate cancer versus benign prostatic hyperplasia and for the evaluation of the disease aggressiveness.

\section{Urine biomarkers}

- Among urine biomarkers, sarcosine, prostate cancer antigen 3 (PCA3) and the TMPRSS2:ERG gene fusion have a promising diagnostic potential.

- Sarcosine is an $\mathrm{N}$-methyl derivative of glycine that was significantly increased during prostate cancer progression from normal through localized to metastatic disease. Urinary sarcosine was found to be significantly higher in prostate cancer patients than in controls.

- Sarcosine can be measured both in the urine and in the serum. Serum sarcosine had a higher predictive value than total PSA and free PSA in detecting prostate cancer in patients with total serum PSA $<4 \mathrm{ng} / \mathrm{ml}$. In addition, elevated circulating sarcosine levels have been showed in patients with metastatic castration-resistant prostate cancer compared with patients with nonmetastatic disease.

- Sarcosin may be employed in combination with PCA3. The PCA3 gene is a noncoding RNA mapped to chromosome 9q21-22. Its usefulness relies in its ability to predict re-biopsy outcome in patients with and without either high-grade prostatic intraepithelial neoplasia or atypical small acinar proliferation.

- The TMPRSS2:ERG gene fusion is a frequent chromosomal rearrangement in prostate cancer. It is the result of the fusion of the transmembrane protease, serine 2 (TMPRSS2) gene and the v-ets avian erythroblastosis virus E26 oncogene homolog (ERG) gene or other ETS (E26 transformation specific) transcription factors. TMPRSS2:ERG is highly specific for predicting clinically significant prostate cancer on biopsy, despite the relatively low sensitivity.

\section{Tissue biomarkers}

- Prolaris (Myriad Genetics, UT, USA) is a commercially available test that evaluates the expression of 31 cell-cycle progression genes, normalized to 15 housekeeper genes. One study of 413 prostatectomized men reported a hazard ratio for recurrence for each unit increase in the Prolaris score of $1.7(95 \% \mathrm{Cl}: 1.3-2.4)$, after adjusting for a standard postoperative risk assessment (Cancer of the Prostate Risk Assessment Postsurgical).

- The Oncotype DX ${ }^{\circledR}$ Genomic Prostate Score test (Genomic Health, Inc., CA, USA) is a RT-PCR-based assay that evaluates the expression of 17 genes. It is able to provide prognostic information and assess the risk of adverse pathology, defined as primary Gleason pattern 4 or any pattern 5 and/or pT3 disease, after radical prostatectomy.

- Hypermethylation involving specific gene promoters in the context of a generalized hypomethylation can cause silencing of tumor suppressor genes and thus promote tumor growth.

- In the MATLOC study, histopathologically negative prostate biopsy samples with matching subsequent either positive (cases) or negative (controls) repeat biopsy samples taken within 30 months were analyzed via a quantitative methylation specific PCR assay panel that included GSTP1, APC and RASSF1. This epigenetic assay yielded a negative predictive value of $90 \%$ (95\% Cl: $87-93)$. 


\section{EXECUTIVE SUMMARY (CONT.)}

\section{Tissue biomarkers (cont.)}

- Recently, promising prognostic methylation marker candidates have been identified, the most prominent being PITX2, GABRE miR-452 miR-224, C1orf114 and the marker panel AOX1/C1orf114/HAPLN3 which have all been reported as independent predictors of biochemical recurrence after radical prostatectomy.

\section{Conclusion}

- Prostate cancer prognosis is highly heterogeneous, with men harboring an indolent disease that can be safely observed and men whose disease is rapidly fatal.

- The widespread use of PSA as a screening tool has contributed to improve early detection and reduce mortality, but it has also been responsible for overdiagnosis and overtreatment.

\section{Future perspective}

- The possibility for the combined use of novel biomarker assays to optimize accuracy is intriguing, but must be weighed against increased costs.

- There is a compelling need not only for additional well-designed, large, multicenter, prospective trials, but also for economic studies evaluating the cost/benefit ratio of novel biomarkers.

- Increased attention to reimbursement policies is also required in order to maximize their impact in clinical practice.

\section{References}

Papers of special note have been highlighted as:

- of interest; $\bullet$ of considerable interest

1 Schroder FH, Hugosson J, Roobol MJ et al. Screening and prostate cancer mortality: results of the European Randomised Study of Screening for Prostate Cancer (ERSPC) at 13 years of follow-up. Lancet 384(9959), 2027-2035 (2014).

2 Heijnsdijk EA, Wever EM, Auvinen A et al. Quality-of-life effects of prostate-specific antigen screening. N. Engl. J. Med. 367(7), 595-605 (2012).

3 Heijnsdijk EA, De Carvalho TM, Auvinen A et al. Cost-effectiveness of prostate cancer screening: a simulation study based on ERSPC data. J. Natl Cancer Inst. 107(1), 366 (2015).

4 Morgan T, Palapattu G, Wei J. Screening for prostate cancer-beyond total PSA, utilization of novel biomarkers. Curr. Urol. Rep. 16(9), 537 (2015).

5 Louie KS, Seigneurin A, Cathcart P, Sasieni P. Do prostate cancer risk models improve the predictive accuracy of PSA screening? A meta-analysis. Ann. Oncol. 26(5), 848-864 (2015).

6 Ankerst DP, Hoefler J, Bock S et al. Prostate Cancer Prevention Trial Risk Calculator 2.0 for the prediction of low- vs high-grade prostate cancer. Urology 83(6), 1362-1367 (2014).

7 Tefekli A, Tunc M. Future prospects in the diagnosis and management of localized prostate cancer. ScientificWorldJournal 2013, 347263 (2013).
8 Lilja H. A kallikrein-like serine protease in prostatic fluid cleaves the predominant seminal vesicle protein. J. Clin. Invest. 76(5), 1899-1903 (1985).

9 Catalona WJ, Smith DS, Ratliff TL et al. Measurement of prostate-specific antigen in serum as a screening test for prostate cancer. N. Engl. J. Med. 324(17), 1156-1161 (1991).

10 Brawer MK. Prostate-specific antigen: current status. CA Cancer J. Clin. 49(5), 264-281 (1999).

11 Vickers AJ, Cronin AM, Aus G et al. A panel of kallikrein markers can reduce unnecessary biopsy for prostate cancer: data from the European Randomized Study of Prostate Cancer Screening in Goteborg, Sweden. BMC Med. 619 (2008).

12 Mcvary KT. BPH: epidemiology and comorbidities. Am. J. Manag. Care 12(Suppl. 5), S122-S128 (2006).

13 Schroder FH, Carter HB, Wolters T et al. Early detection of prostate cancer in 2007. Part 1: PSA and PSA kinetics. Eur. Urol. 53(3), 468-477 (2008).

14 Moul JW, Sesterhenn IA, Connelly RR et al. Prostate-specific antigen values at the time of prostate cancer diagnosis in AfricanAmerican men. JAMA 274(16), 1277-1281 (1995).

15 Oesterling JE, Jacobsen SJ, Chute CG et al. Serum prostate-specific antigen in a community-based population of healthy men. Establishment of age-specific reference ranges. JAMA 270 (7), 860-864 (1993).
16 Carter HB, Albertsen PC, Barry MJ et al. Early detection of prostate cancer: AUA Guideline. J. Urol. 190(2), 419-426 (2013).

17 Savblom C, Hallden C, Cronin AM et al. Genetic variation in KLK2 and KLK3 is associated with concentrations of hK2 and PSA in serum and seminal plasma in young men. Clin. Chem. 60(3), 490-499 (2014).

18 Kundu SD, Roehl KA, Yu X, Antenor JA, Suarez BK, Catalona WJ. Prostate specific antigen density correlates with features of prostate cancer aggressiveness. J. Urol. 177(2), 505-509 (2007).

19 Radwan MH, Yan Y, Luly JR et al. Prostatespecific antigen density predicts adverse pathology and increased risk of biochemical failure. Urology 69 (6), 1121-1127 (2007).

20 Corcoran NM, Casey RG, Hong MK et al. The ability of prostate-specific antigen (PSA) density to predict an upgrade in Gleason score between initial prostate biopsy and prostatectomy diminishes with increasing tumour grade due to reduced PSA secretion per unit tumour volume. BJU Int. 110(1), 36-42 (2012).

21 Magheli A, Hinz S, Hege C et al. Prostate specific antigen density to predict prostate cancer upgrading in a contemporary radical prostatectomy series: a single center experience. J. Urol. 183(1), 126-131 (2010).

22 Venderbos LD, Roobol MJ, Bangma CH et al. Rule-based versus probabilistic selection for active surveillance using three definitions of insignificant prostate cancer. World J. Urol. doi:10.1007/s00345-015-1628-y (2015) (Epub ahead of print). 
23 Vickers AJ, Savage C, O'brien MF, Lilja H. Systematic review of pretreatment prostatespecific antigen velocity and doubling time as predictors for prostate cancer. J. Clin. Oncol. 27(3), 398-403 (2009).

- This meta-analysis shows that there is insufficient evidence proving that calculation of prostate-specific antigen velocity or doubling time in untreated patients provides additional information with respect to that provided by absolute prostate-specific antigen level alone.

24 Vickers AJ, Till C, Tangen CM, Lilja H, Thompson IM. An empirical evaluation of guidelines on prostate-specific antigen velocity in prostate cancer detection. J. Natl. Cancer Inst. 103(6), 462-469 (2011).

25 Carter HB, Kettermann A, Ferrucci L, Landis P, Metter EJ. Prostate-specific antigen velocity risk count assessment: a new concept for detection of life-threatening prostate cancer during window of curability. Urology 70 (4), 685-690 (2007).

26 Tosoian JJ, Loeb S, Feng Z et al. Association of [-2]proPSA with biopsy reclassification during active surveillance for prostate cancer. J. Urol. 188(4), 1131-1136 (2012).

27 Patel HD, Feng Z, Landis P, Trock BJ, Epstein JI, Carter HB. Prostate specific antigen velocity risk count predicts biopsy reclassification for men with very low risk prostate cancer. J. Urol. 191(3), 629-637 (2014).

28 Khan MA, Carter HB, Epstein JI et al. Can prostate specific antigen derivatives and pathological parameters predict significant change in expectant management criteria for prostate cancer? J. Urol. 170 (6 Pt 1), 2274-2278 (2003).

29 Van As NJ, Norman AR, Thomas K et al. Predicting the probability of deferred radical treatment for localised prostate cancer managed by active surveillance. Eur. Urol. 54(6), 1297-1305 (2008).

$30 \mathrm{Ng}$ MK, Van As N, Thomas K et al. Prostate-specific antigen (PSA) kinetics in untreated, localized prostate cancer: PSA velocity vs PSA doubling time. BJU Int. 103(7), 872-876 (2009).

31 Vasarainen H, Salman J, Salminen H et al. Predictive role of free prostate-specific antigen in a prospective active surveillance program (PRIAS). World J. Urol. 33(11), 1735-1740 (2015).

32 Hirama H, Sugimoto M, Ito K, Shiraishi T, Kakehi Y. The impact of baseline [-2] proPSA-related indices on the prediction of pathological reclassification at 1 year during active surveillance for low-risk prostate cancer: the Japanese multicenter study cohort. J. Cancer Res. Clin. Oncol. 140(2), 257-263 (2014).

33 Mikolajczyk SD, Millar LS, Wang TJ et al. A precursor form of prostate-specific antigen is more highly elevated in prostate cancer compared with benign transition zone prostate tissue. Cancer Res. 60 (3), 756-759 (2000).

- This article is of considerable interest in that it reports about the potential diagnostic value of a precursor form of prostate-specific antigen.

34 Mikolajczyk SD, Marker KM, Millar LS et al. A truncated precursor form of prostatespecific antigen is a more specific serum marker of prostate cancer. Cancer Res. 61(18), 6958-6963 (2001).

35 Jansen FH, Van Schaik RH, Kurstjens J et al. Prostate-specific antigen (PSA) isoform p2PSA in combination with total PSA and free PSA improves diagnostic accuracy in prostate cancer detection. Eur. Urol. 57(6), 921-927 (2010).

36 Sokoll LJ, Sanda MG, Feng Z et al. A prospective, multicenter, National Cancer Institute Early Detection Research Network study of [-2]proPSA: improving prostate cancer detection and correlating with cancer aggressiveness. Cancer Epidemiol. Biomarkers Prev. 19(5), 1193-1200 (2010).

37 Lazzeri M, Haese A, Abrate A et al. Clinical performance of serum prostate-specific antigen isoform [-2]proPSA (p2PSA) and its derivatives, $\%$ p2PSA and the prostate health index (PHI), in men with a family history of prostate cancer: results from a multicentre European study, the PROMEtheuS project. BJU Int. 112(3), 313-321 (2013).

38 Lazzeri M, Abrate A, Lughezzani G et al. Relationship of chronic histologic prostatic inflammation in biopsy specimens with serum isoform [-2]proPSA (p2PSA),\%p2PSA, and prostate health index in men with a total prostate-specific antigen of $4-10 \mathrm{ng} / \mathrm{ml}$ and normal digital rectal examination. Urology 83(3), 606-612 (2014).

39 Bruzzese D, Mazzarella C, Ferro M et al. Prostate health index vs percent free prostate-specific antigen for prostate cancer detection in men with "gray" prostate-specific antigen levels at first biopsy: systematic review and meta-analysis. Transl. Res. 164(6), 444-451 (2014).

40 Haese A, Graefen M, Steuber T et al. Human glandular kallikrein 2 levels in serum for discrimination of pathologically organconfined from locally-advanced prostate cancer in total PSA-levels below $10 \mathrm{ng} / \mathrm{ml}$. Prostate 49(2), 101-109 (2001).

41 Gupta A, Roobol MJ, Savage CJ et al. A four-kallikrein panel for the prediction of repeat prostate biopsy: data from the European Randomized Study of Prostate Cancer screening in Rotterdam, Netherlands. Br. J. Cancer 103(5), 708-714 (2010).

42 Bryant RJ, Sjoberg DD, Vickers AJ et al. Predicting high-grade cancer at ten-core prostate biopsy using four kallikrein markers measured in blood in the ProtecT study. J. Natl. Cancer Inst. 107(7), (2015).

-. This article is of considerable interest in that it proved the predictive value of four kallikrein markers pathologically insignificant compared with aggressive prostate cancer in radical prostatectomy specimens.

43 Carlsson S, Maschino A, Schroder F et al. Predictive value of four kallikrein markers for pathologically insignificant compared with aggressive prostate cancer in radical prostatectomy specimens: results from the European Randomized Study of Screening for Prostate Cancer section Rotterdam. Eur. Urol. 64(5), 693-699 (2013).

44 Benchikh A, Savage C, Cronin A et al. A panel of kallikrein markers can predict outcome of prostate biopsy following clinical work-up: an independent validation study from the European Randomized Study of Prostate Cancer screening, France. BMC Cancer 10635 (2010).

45 Parekh DJ, Punnen S, Sjoberg DD et al. A multi-institutional prospective trial in the USA confirms that the 4 Kscore accurately identifies men with high-grade prostate cancer. Eur. Urol. doi:10.1016/j. eururo.2014.10.021 (2014) (Epub ahead of print).

46 Stattin P, Vickers AJ, Sjoberg DD et al. Improving the specificity of screening for lethal prostate cancer using prostate-specific antigen and a panel of kallikrein markers: a nested case-control study. Eur. Urol. 68(2), 207-213 (2015).

47 Bartel DP. MicroRNAs: genomics, biogenesis, mechanism, and function. Cell 116(2), 281-297 (2004).

48 Casanova-Salas I, Rubio-Briones J, Fernandez-Serra A, Lopez-Guerrero JA. miRNAs as biomarkers in prostate cancer. Clin. Transl. Oncol. 14(11), 803-811 (2012).

49 Penney KL, Sinnott JA, Fall K et al. mRNA expression signature of Gleason grade predicts lethal prostate cancer. J. Clin. Oncol. 29(17), 2391-2396 (2011). 
50 Ren Q, Liang J, Wei J et al. Epithelial and stromal expression of miRNAs during prostate cancer progression. Am. J. Transl. Res. 6(4), 329-339 (2014).

51 Lichner Z, Ding Q, Samaan S et al. miRNAs dysregulated in association with Gleason grade regulate extracellular matrix, cytoskeleton and androgen receptor pathways. J. Pathol. doi: 10.1002/path.4568 (2015) (Epub ahead of print).

52 Mahn R, Heukamp LC, Rogenhofer S, Von Ruecker A, Muller SC, Ellinger J. Circulating microRNAs (miRNA) in serum of patients with prostate cancer. Urology 77(5), 1265 e1269-1265 e1216 (2011).

53 Shen J, Hruby GW, Mckiernan JM et al. Dysregulation of circulating microRNAs and prediction of aggressive prostate cancer. Prostate 72(13), 1469-1477 (2012).

54 Lucarelli G, Rutigliano M, Galleggiante V et al. Metabolomic profiling for the identification of novel diagnostic markers in prostate cancer. Expert Rev. Mol. Diagn. 15(9), 1211-224 (2015).

55 Sreekumar A, Poisson LM, Rajendiran TM et al. Metabolomic profiles delineate potential role for sarcosine in prostate cancer progression. Nature 457(7231), 910-914 (2009).

56 Melling N, Thomsen E, Tsourlakis MC et al. Overexpression of Enhancer of zeste homolog 2 (EZH2) characterizes an aggressive subset of prostate cancers and predicts patient prognosis independently from pre- and postoperatively assessed clinicopathological parameters. Carcinogenesis 36(11), 1333-1340 (2015).

57 Cao DL, Ye DW, Zhu Y, Zhang HL, Wang YX, Yao XD. Efforts to resolve the contradictions in early diagnosis of prostate cancer: a comparison of different algorithms of sarcosine in urine. Prostate Cancer Prostatic Dis. 14(2), 166-172 (2011).

58 Lucarelli G, Fanelli M, Larocca AM et al. Serum sarcosine increases the accuracy of prostate cancer detection in patients with total serum PSA less than $4.0 \mathrm{ng} / \mathrm{ml}$. Prostate 72(15), 1611-1621 (2012).

59 Lucarelli G, Rutigliano M, Bettocchi C et al. Spondin-2, a secreted extracellular matrix protein, is a novel diagnostic biomarker for prostate cancer. J. Urol. 190(6), 2271-2277 (2013).

60 Ferro M, Lucarelli G, Bruzzese D et al. Improving the prediction of pathologic outcomes in patients undergoing radical prostatectomy: the value of prostate cancer antigen 3 (PCA3), prostate health index (phi) and sarcosine. Anticancer Res. 35(2), 1017-1023 (2015).
61 Lucarelli G, Ditonno P, Bettocchi C et al. Serum sarcosine is a risk factor for progression and survival in patients with metastatic castration-resistant prostate cancer. Future Oncol. 9(6), 899-907 (2013).

62 Koutros S, Meyer TE, Fox SD et al. Prospective evaluation of serum sarcosine and risk of prostate cancer in the Prostate, Lung, Colorectal and Ovarian Cancer Screening Trial. Carcinogenesis 34(10), 2281-2285 (2013).

63 Bussemakers MJ, Van Bokhoven A, Verhaegh GW et al. DD3: a new prostate-specific gene, highly overexpressed in prostate cancer. Cancer Res. 59(23), 5975-5979 (1999).

64 De Kok JB, Verhaegh GW, Roelofs RW et al. DD3(PCA3), a very sensitive and specific marker to detect prostate tumors. Cancer Res. 62(9), 2695-2698 (2002).

65 Luo Y, Gou X, Huang P, Mou C. The PCA3 test for guiding repeat biopsy of prostate cancer and its cut-off score: a systematic review and meta-analysis. Asian J. Androl. 16(3), 487-492 (2014).

66 Hessels D, Smit FP, Verhaegh GW, Witjes JA, Cornel EB, Schalken JA. Detection of TMPRSS2-ERG fusion transcripts and prostate cancer antigen 3 in urinary sediments may improve diagnosis of prostate cancer. Clin. Cancer Res. 13(17), 5103-5108 (2007).

67 Mehra R, Han B, Tomlins SA et al. Heterogeneity of TMPRSS2 gene rearrangements in multifocal prostate adenocarcinoma: molecular evidence for an independent group of diseases. Cancer Res. 67(17), 7991-7995 (2007).

68 Magi-Galluzzi C, Tsusuki T, Elson P et al. TMPRSS2-ERG gene fusion prevalence and class are significantly different in prostate cancer of Caucasian, African-American and Japanese patients. Prostate 71(5), 489-497 (2011).

69 Robert G, Jannink S, Smit F et al. Rational basis for the combination of PCA 3 and TMPRSS2:ERG gene fusion for prostate cancer diagnosis. Prostate 73(2), 113-120 (2013).

70 Laxman B, Morris DS, Yu J et al. A first-generation multiplex biomarker analysis of urine for the early detection of prostate cancer. Cancer Res. 68(3), 645-649 (2008).

71 Salami SS, Schmidt F, Laxman B et al. Combining urinary detection of TMPRSS2:ERG and PCA3 with serum PSA to predict diagnosis of prostate cancer. Urol. Oncol. 31(5), 566-571 (2013).

72 Cao DL, Ye DW, Zhang HL, Zhu Y, Wang YX, Yao XD. A multiplex model of combining gene-based, protein-based, and metabolitebased with positive and negative markers in urine for the early diagnosis of prostate cancer. Prostate 71(7), 700-710 (2011).

73 Cooperberg MR, Hilton JF, Carroll PR. The CAPRA-S score: a straightforward tool for improved prediction of outcomes after radical prostatectomy. Cancer 117(22), 5039-5046 (2011).

74 Cuzick J, Berney DM, Fisher G et al. Prognostic value of a cell cycle progression signature for prostate cancer death in a conservatively managed needle biopsy cohort. Br. J. Cancer 106(6), 1095-1099 (2012).

75 Cooperberg MR, Simko JP, Cowan JE et al. Validation of a cell-cycle progression gene panel to improve risk stratification in a contemporary prostatectomy cohort. J. Clin. Oncol. 31(11), 1428-1434 (2013).

76 Klein EA, Cooperberg MR, Magi-Galluzzi C et al. A 17-gene assay to predict prostate cancer aggressiveness in the context of Gleason grade heterogeneity, tumor multifocality, and biopsy undersampling. Eur. Urol. 66(3), 550-560 (2014).

77 Cullen J, Rosner IL, Brand TC et al. A Biopsy-based 17-gene genomic prostate score predicts recurrence after radical prostatectomy and adverse surgical pathology in a racially diverse population of men with clinically low- and intermediaterisk prostate cancer. Eur. Urol. 68(1), 123-131 (2015).

- This article is of interest in that it showed that the Genomic Prostate Score can evaluate the aggressiveness of the cancer.

78 Heichman KA, Warren JD. DNA methylation biomarkers and their utility for solid cancer diagnostics. Clin. Chem. Lab. Med. 50(10), 1707-1721 (2012).

79 Desotelle J, Truong M, Ewald J et al. CpG island hypermethylation frequently silences FILIP1L isoform 2 expression in prostate cancer. J. Urol. 189(1), 329-335 (2013).

80 Lee WH, Morton RA, Epstein JI et al. Cytidine methylation of regulatory sequences near the pi-class glutathione $S$-transferase gene accompanies human prostatic carcinogenesis. Proc. Natl Acad. Sci. USA 91(24), 11733-11737 (1994).

81 Stewart GD, Van Neste L, Delvenne P et al. Clinical utility of an epigenetic assay to detect occult prostate cancer in histopathologically negative biopsies: results of the MATLOC study. J. Urol. 189(3), 1110-1116 (2013).

82 Partin AW, Van Neste L, Klein EA et al. Clinical validation of an epigenetic assay to 
predict negative histopathological results in repeat prostate biopsies. J. Urol. 192(4), 1081-1087 (2014).

83 Litovkin K, Van Eynde A, Joniau S et al. DNA methylation-guided prediction of clinical failure in high-risk prostate cancer. PLoS ONE 10(6), e0130651 (2015).

84 Daniunaite K, Jarmalaite S, Kalinauskaite N et al. Prognostic value of RASSF1 promoter methylation in prostate cancer. J. Urol. 192(6), 1849-1855 (2014).
85 Strand SH, Orntoft TF, Sorensen KD. Prognostic DNA methylation markers for prostate cancer. Int. J. Mol. Sci. 15(9), 16544-16576 (2014).

86 Robinson D, Van Allen EM, Wu YM et al. Integrative clinical genomics of advanced prostate cancer. Cell 161(5), 1215-1228 (2015).

87 Barbieri CE, Baca SC, Lawrence MS et al. Exome sequencing identifies recurrent $S P O P$, FOXA1 and MED12 mutations in prostate cancer. Nat. Genet. 44(6), 685-689 (2012).
88 Beltran H, Yelensky R, Frampton GM et al. Targeted next-generation sequencing of advanced prostate cancer identifies potential therapeutic targets and disease heterogeneity. Eur. Urol. 63(5), 920-926 (2013).

89 Bancroft EK, Page EC, Castro E et al. Targeted prostate cancer screening in $B R C A 1$ and $B R C A 2$ mutation carriers: results from the initial screening round of the IMPACT study. Eur. Urol. 66(3), 489-499 (2014). 